\title{
Gorillas with white sclera: a naturally occurring variation in a morphological trait linked to social cognitive functions
}

\author{
Jessica A. Mayhew ${ }^{1,2}$ and Juan-Carlos Gómez ${ }^{1}$ \\ ${ }^{1}$ School of Psychology and Neuroscience, University of St. Andrews, St. Andrews, Fife, \\ KY16 9JP, Scotland, UK \\ ${ }^{2}$ Anthropology and Museum Studies, Central Washington University, 400 E. University \\ Way, MS 7544, Ellensburg, WA, 98926-7519 \\ mayhewj@cwu.edu (J. Mayhew) \\ jg5@st-andrews.ac.uk (J-C Gómez)
}

*Corresponding Author:

Juan-Carlos Gómez

E-mail: jg5@st-andrews.ac.uk

Telephone: +01334 462059

Short title: Naturally occurring white sclera in gorillas

To be published in:

American Journal of Primatology, 2015. 


\begin{abstract}
Human eye morphology is considered unique among the primates in that humans possess larger width/height ratios (WHR), expose a greater amount of visible sclera (SSI; width of exposed eyeball/width of visible iris), and critically, have a white sclera due to a lack of pigmentation. White sclera in humans amplifies gaze direction, whereas the alldark eyes of apes are hypothesized to conceal gaze from others. This study examines WHR and SSI in humans $(N=13)$ and gorillas $(N=85)$ engaged in direct and averted gazes and introduces a qualitative assessment of sclera color to evaluate variations in sclera pigmentation. The results confirm previous findings that humans possess a larger WHR than gorillas but indicate that humans and gorillas display similar amounts of visible sclera. Additionally, $72 \%(N=124)$ of gorilla eyes in this sample deviated from the assumed all-dark eye condition. This questions whether gaze-camouflage is the primary function of darkened sclera in non-human primates or whether other functional roles can be ascribed to the sclera, light or dark. We argue that white sclera evolved to amplify direct gazes in humans, which would have played a significant role in the development of ostensive communication, which is communication that both shows something and shows the intention to show something. We conclude that the horizontal elongation of the human eye, rather than sclera color, more reliably distinguishes human from great ape eyes, represented here by gorillas.
\end{abstract}

Keywords: White sclera; gaze camouflaging; gorillas; eye morphology; gaze following.

\title{
INTRODUCTION
}

Much of the non-human primate coloration literature examines pelage and skin color with particular emphasis on its relationship to sexual selection, concealment, aggression, and dominance levels [Caro, 2005; Caro, 2009]. In primates, color changes can occur with sexual maturation, e.g., male gorillas (Gorilla gorilla) experience a silvering of the hair on the back indicative of adulthood, or some primate infants begin life with a conspicuous natal coat that becomes less dramatic with age, e.g., the orange coat of infant Silvered Leaf monkeys (Trachypithecus cristatus) [Alley, 1980; Treves, 1997; Bradley and Mundy, 2008]. Other bright and highly visible colors often occur on the bare-skinned areas of both males and females, e.g., the bright red sexual swellings of 
female Hamadryas baboons (Papio hamadryas) in estrus, the bright blue genitals of male Vervet monkeys (Chlorocebus pygerythrus), and the red- and blue-banded face of the male Mandrill (Mandrillus sphinx). The adaptive significance of such bright colors is suggested to relate to the emergence of trichromatic vision in primates, where trichromacy was selected to detect these biologically driven, socially significant color cues [Changizi et al., 2006].

There are brief mentions of sclera color in some natural history accounts of primates; for example, Schaller remarks that in mountain gorillas, white is only visible in the dark brown eye when it is rolled to one side [Schaller, 1963, p. 71]. However, comprehensive evaluations of non-human primate eye color remain limited [e.g., iris color in Macaca fuscata: Zhang and Watanabe, 2007], and there are few studies that investigate sclera color or eye morphology [e.g., Schmittbuhl et al., 1999; Kobayashi and Kohshima, 2001; Liang et al., 2005; Knapp et al., 2007; Kobayashi and Hashiya, 2011].

To date, Kobayashi and Kohshima (2001) (hereafter abbreviated KK) is the only study that systematically differentiates and categorizes extant primate eye coloration. The authors used both quantitative (width/height ratios, WHR, and exposed sclera size indices, SSI) and qualitative measures (color comparisons of the iris, sclera, and surrounding facial skin) and determined that the morphological characteristics of the modern human eye are unique among extant primates. In this study, KK determined that human eyes are more horizontally elongated compared to non-human primates, and direct gazes expose a greater amount of visible white sclera. The authors suggest that these features of the human eye might be an adaptation to enhance the salience and detectability of eye gaze direction as a source of social information and possibly developed because of decreased predation pressures due to increasing body size and terrestriality.

$\mathrm{KK}$ also noted that great ape eyes are all-dark with a dark iris and sclera, which contrasts with the dark iris/white sclera combination observed in humans. Histological evidence has established that the white sclera of humans results from a transparent conjunctiva and the absence of pigmentation. The non-human primate evidence from KK indicates that primates also possess a white inner sclera layer with pigmentation deposits in the epithelium cornea, conjunctiva, and sclera [Kobayashi and Kohshima, 2001; for gorillas, see Knapp et al., 2007]. These color schemes put forth in the KK study are often noted as a key difference between humans and other primates, particularly in comparative gaze-following research [e.g., Emery, 2000; Laube et al., 2011]. White sclera has been suggested to be a human adaptation to create a "cooperative eye" that enhances gaze signaling between communicating individuals to facilitate joint attention, particularly during joint interactions [the cooperative eye hypothesis: Tomasello et al., 2007]. Therefore, the evolution of white sclera would be a uniquely human adaptation linked to uniquely human communicative functions [Kobayashi and Kohshima, 2001; Tomasello et al., 2007]. The inverse reasoning has been used for the absence of white sclera in other primates. All-dark eyes possibly confer other primates a different gaze advantage, namely, gaze camouflage. The advantages of concealing gaze direction from conspecifics have been discussed mainly relative to competitive contexts: the concealment of eye gaze direction could aid in the avoidance of conspecific aggression or deceive conspecifics about interesting objects within the environment [Kobayashi and Kohshima, 2001; Tomasello et al., 2007]. However, little research has been conducted to 
investigate this hypothesis further.

It is important to note that not all humans possess white sclera, and some human groups may develop scleral spots (e.g., Eskimos [Norn, 1985]) or have transient or permanent color changes (e.g., red from crying, temporary pathology or irritation, drug use, etc.[Provine et al., 2011]; yellow showcasing liver disease or aging [Provine et al., $2013 b]$ ). Individuals with yellow-tinted sclera are perceived as unhealthy, unattractive, and older. Similarly, individuals with red sclera are perceived as sad, unhealthy, and unattractive [Provine et al., 2013b]. The results are consistent for both monocular and binocular redness, with binocular redness generating a stronger effect than monocular stimuli, which may relate to the use of symmetry as an indicator of desirable traits during mate selection [Little et al., 2008; Provine et al., 2013a]. These results suggest that white sclera in humans is an honest signal of health, attractiveness, and age [Provine et al., 2013a; Provine et al., 2013b]. Overall, these results illustrate that the sclera is a flexible medium for communication, and humans are sensitive to changes in its color, which may provide important social (e.g., emotion) and/or biological information (e.g., health) to observers.

From KK's research, all great ape eyes are expected to follow the all-dark color scheme. However, casual observations of many great apes, particularly gorillas (although not restricted to them), suggest that a uniformly dark eye may not be the universal rule, and instead, degrees of variation may occur with relative frequency (Fig. 1). Indeed, in chimpanzees, Goodall reported the presence of white sclera in two adult males from Gombe, of which one had completely white sclera as seen in humans [Goodall, 1986, p. 72]. Goodall noted that this male always appeared to be vigilant because the obvious contrast between the white sclera and dark surrounding face drew attention to his eye movement [Goodall, 1986, p. 121].

Insert Fig. 1 here

The visible sclera of some gorillas may be much lighter than either the iris or surrounding facial skin, particularly with an averted gaze. Sclera depigmentation can occur to a "human-like" degree but does not have to occur symmetrically and may be heavier in one eye. Therefore, in this paper, we aimed to investigate the incidence and extent of the white sclera trait in gorillas. Using the methods developed by KK, we expanded on the original results using a larger sample of individuals engaged in direct and averted gazes and added a graded measurement of the degree of sclera depigmentation.

\section{METHODS}

\section{Study sample}

Eighty-five front face photographs of western lowland (Gorilla gorilla gorilla, $N$ $=60$ ) and mountain gorilla (Gorilla beringei beringei, $\mathrm{N}=25$ ) faces were collected from various resources: Google images $(N=27)$, known zoo-housed individuals contributed by the Paignton and Belfast zoos (K. Pullen, Paignton Zoo; J. Mansell, Belfast Zoo; $N=$ $10)$ or zoo websites $(N=32)$, and a book documenting mountain gorilla group members (Eckhart and Lanjouw, 2008; $N=16$ ). Two photographs of 13 human faces engaged in direct and averted gazes ( $N=26$ photographs) were also collected as a control comparison. The photograph location, name of the individual within the photograph, and species were obtained to avoid repeated analysis. Gaze direction, relative to the camera, 
was also recorded as direct (looking directly into the camera, $N=27$ ) or averted $(N=58)$. The research presented here used photographs and was thus exempt from Institutional Animal Care Committee review. This research adhered to the legal requirements of the country in which it was conducted and to the American Society of Primatologists' Principles for the Ethical Treatment of Primates.

\section{Measurements}

WHR and SSI. Each photograph was analyzed using the NIH ImageJ program $(1.45 \mathrm{o}, \mathrm{W}$. Rasband, National Institutes of Health, USA, http://imagej.nih.gov/ij) measuring width/height ratio (WHR) and exposed sclera size index (SSI) for the left and right eyes similar to the methods of KK (Fig. 2). WHR was A/B, where $\mathrm{A}=$ the distance between the corners of the eye, including the vestigial portion of the nictitating membrane in the corner of the eye, and $\mathrm{B}=$ the longest perpendicular line between the top and bottom eyelids. WHR measures the relative elongation of the eye. SSI was C/D, where $\mathrm{C}=$ the width of the exposed eyeball, excluding the vestigial portion of the nictitating membrane in the corner of the eye, and D = the width of the visible iris. SSI measures the amount of visible sclera within the eyeball.

Insert Fig. 2 here

Both measurements estimate the size of the contrast between the sclera and iris. For each photograph, A-D were measured three times for each visible eye, and the values were then averaged. The left and right eye values were then averaged to generate an overall WHR and SSI for each photograph, which were then further combined to generate mean WHR and SSI for direct and averted gazes categorized by species.

Sclera color. KK proposed a relative graded scale between face, sclera, and iris color for primates: 1) face $=$ sclera $=$ iris; 2) face $<$ sclera $=$ iris; 3) face $=$ sclera $>$ iris; and 4) face $>$ sclera $<$ iris. The preliminary review of our dataset suggested that white sclera might vary across a continuum. Therefore, these classifications were too narrow to represent the variability within this dataset, and we introduced two new measurements to quantify 1) the proportion of the eye that contained whitened sclera and 2) the pigment degradation pattern. No previous study has generated guidelines for such an empirical analysis; therefore, we used a $0-10$ scale, which corresponded with the coder's assessment of the amount of visible, lightened sclera. For example, if the eye contained no visibly lightened sclera, it was scored a 0 , whereas an eye with a completely white sclera was scored a 10. Additionally, we defined six qualitative white sclera patterns to further categorize and represent depigmentation in the eye (Fig. 3): 1) all-dark, the sclera is completely dark, and there is no visible depigmentation; 2) patchy, areas of variable sizes are lighter; 3) banded, the lightened sclera appears to run from the top to the bottom eyelid in a column and is often (but not always) positioned between two darker areas of sclera; 4) crescent, the lightened sclera forms a half-moon shape, in which there is less depigmentation around the middle edge of the iris (darker region) and more depigmentation at the iris poles (lighter regions); 5) combination, two of the above patterns are present; or 6) all-white, the sclera is completely lightened.

\section{RESULTS}

Insert Fig. 3 here

Two coders independently scored gaze direction and sclera coloration to assess 
inter-rater reliability (IRR). The IRR for gaze direction was $k=0.83$, showing a strong agreement between the two coders $(P<0.001)$. The IRR for sclera coloration was rated as being in substantial agreement with a Spearman's correlation of $r=0.98(P<0.001)$ with $\leq 1$ degree of agreement in $93.7 \%$ of responses. The IRR for the white sclera patterns was in strong agreement with a Spearman's correlation of $r=0.92(P<0.001)$. All $P$-values are reported at a 0.05 significance level.

\section{Direct gaze}

The direct gazing mountain and lowland gorilla mean WHR $(1.86 \pm 0.29, N=8$ and $2.02 \pm 0.29, N=21$, respectively; independent samples $t$-test: $t=-1.33, \mathrm{df}=27, P>$ $0.05)$ and SSI (1.67 \pm 0.25 and $1.64 \pm 0.35$, respectively; $t=0.14, \mathrm{df}=22, P>0.05)$ were not significantly different. Therefore, the species data were combined and compared to KK's gorilla results. The mean direct gaze WHR of gorillas overall $(1.98 \pm 0.29$, range $=$ 1.4-2.7, $N=29)$ was consistent with the value observed in the original study $(2.0$, range = $1.8-2.1, N=4)$. The mean direct gaze SSI of gorillas in this study $(1.65 \pm 0.32$, range $=$ $1.2-2.4, N=24)$ was also consistent with the original results $(1.7$, range $=1.5-1.9, N=4)$. The mean direct gaze WHR of humans was $3.24 \pm 0.49$ (range $=2.6-4.4, N=13$ ), which was larger but roughly consistent with the range observed in the original study $(2.9$, range $=2.4-3.2, N=659)$. The mean direct gaze SSI of humans was $1.82 \pm 0.13$ (range $=1.5$ $2.1, N=13)$, which was consistent with the SSI observed in the original study (1.9, range $=1.8-2.1, N=659)$. A significant difference for direct gaze WHR by species was observed (one-way ANOVA: $F=54.61$, df $=2, P<0.001$ ), and a Tukey's $H S D$ determined that the mean of humans was significantly larger $(3.24 \pm 0.49)$ than gorillas (lowland $=2.02 \pm 0.29$, mountain $=1.86 \pm 0.29$ ). No significant differences were observed between species for SSI $(F=1.65, \mathrm{df}=2, P>0.05)$. Overall, the amount of exposed sclera during direct gazes was similar in all three species, but humans had more horizontally elongated eyes.

\section{Averted gaze}

A significant difference was observed for averted gazing humans and mountain and lowland gorilla WHRs $(F=58.57, \mathrm{df}=2, P<0.001)$. Averted gazing humans had a significantly larger mean WHR $(2.94 \pm 0.40, N=13)$ than either lowland or mountain gorillas, and lowland gorillas had a significantly larger WHR $(2.21 \pm 0.28, N=33)$ than mountain gorillas $(1.77 \pm 0.19, N=16)$. No significant species difference was observed for averted SSI $(F=2.38, \mathrm{df}=2, P>0.05)$, although lowland gorillas had the largest value. Overall, the proportion of exposed sclera in averted gazing gorilla and human eyes was comparable. This result was similar to that observed for direct gazes, in which the proportion of exposed sclera was also comparable in all three species.

\section{Direct vs. averted gazes}

The mean WHRs of direct and averted gazing humans were not significantly different $(P=0.10)$. The mean WHRs of direct and averted gazing mountain gorillas were also not significantly different $(P=0.38)$. However, the mean WHR of averted gazing lowland gorillas was significantly larger than their direct gazes $(t=-2.40, \mathrm{df}=52$, $P=0.02$; direct: $\mathrm{WHR}=2.02 \pm 0.29, N=21$; averted: $\mathrm{WHR}=2.21 \pm 0.28, N=33)$. A two-way ANOVA was conducted to examine the effects of gaze direction (direct vs. 
averted) and species (human, mountain gorilla, and lowland gorilla) on WHR. The main effect of gaze direction on WHR was not significant $(F=0.90, \mathrm{df}=1, P=0.34)$.

However, the main effect of species on WHR was significant $(F=110.84, \mathrm{df}=2, P<$ $0.001)$, in which humans possessed larger WHRs than lowland or mountain gorillas, and lowland gorillas possessed larger WHRs than mountain gorillas. The interaction effect of gaze direction and species on WHR was also significant $(F=5.25, \mathrm{df}=2, P=0.007)$, indicating that species had a greater effect than gaze direction.

The mean SSI was larger for averted than direct gazes in all three species and was significantly different in humans $(t=-4.34, \mathrm{df}=24, P<0.001)$ and lowland gorillas $(t=$ $4.34, \mathrm{df}=44, P<0.001)$, thus indicating that the amount of visible sclera for these two species was greater for averted gazes (mountain gorillas were near significance: $t=-1.93$, $\mathrm{df}=20, P=0.067)$. A two-way ANOVA was conducted to examine the effect of gaze direction and species on SSI. There was a significant main effect of gaze direction on SSI $(F=21.98, \mathrm{df}=1, P<0.001)$, which again, indicated that overall, averted gazes had greater SSIs than direct gazes. However, there was no significant main effect of species on SSI $(F=1.04, \mathrm{df}=2, P=0.36)$, which indicated that the overall SSI values (for direct and averted gazes combined) were similar for all three species, and there was no significant interaction effect between gaze direction and species on SSI $(F=2.09, \mathrm{df}=2$, $P=0.13$ ). From this comparison, it appears that humans possessed the greatest WHR for the two gaze types followed by lowland gorillas and mountain gorillas. Additionally, averted gazes displayed more sclera than direct gazes in all three species, but the amount of visible sclera overall was similar in all three species.

Insert Table I here

\section{White sclera proportion and pattern}

Direct and averted gazes in all three species were analyzed to determine the amount of visible depigmentation in the sclera of each eye (Fig. 4). All human eyes in our sample, for both direct and averted gazes, ranked a 10 (all-white) on the $0-10$ scale $(N=$ 52). Two direct gazing mountain gorilla eyes contained depigmentation, but depigmentation in this species was more evident in averted gazing individuals (23 eyes, $57.5 \%$ of eyes). In direct gazing lowland gorilla eyes, $70.0 \%$ (28 eyes) contained depigmentation, and this value increased to $91.0 \%$ (71 eyes) for averted gazes. Typically, direct gazing mountain gorillas were judged to contain similar amounts of depigmentation in each eye $(71.4 \%)$, whereas direct gazing lowland gorillas were often judged to have one eye that contained more depigmentation $(60.0 \%)$. The sclera depigmentation patterns were variable in both gorilla species, particularly when averted gazes were judged (Table II).

$$
\text { Insert Fig. 4, Fig. 5, and Table II here }
$$

\section{DISCUSSION}

This study expanded on Kobayashi and Kohshima (2001) in two manners: 1) it increased the original gorilla sample size from 4 to 85 to re-assess the original quantitative eye measurements and coloration scheme proposed by the authors; and 2) it introduced a comparative evaluation of direct and averted gazes in humans and gorillas. The direct gazing gorilla WHR and SSI values observed in this study support the original study's conclusion that gorilla and human eye morphologies differ; however, our results suggest that the human eye is morphologically different from the gorilla eye in its relative 
elongation (WHR) and not in the proportion of visible sclera to iris (SSI) as was previously suggested. This study indicates that humans and gorillas possess similar amounts of visible sclera, and that the evolutionary change in human eye morphology, at least relative to gorillas, only affects the way in which the iris/sclera combination is displayed, i.e., humans and gorillas display the same amount of sclera, but humans do so within a more horizontally elongated shape. Our results suggest that only the elongation of the eye reliably distinguishes the human from great ape eye (as represented by the gorillas in this study) and not the amount of exposed sclera.

It has been proposed that the elongation of the human eye is an adaptation to a terrestrial lifestyle, in which a larger WHR allows for increased eye movement, thus extending the visual field along the horizontal plane [Kobayashi and Kohshima, 2001]. This increased eye movement would have functional properties for the individual collecting visual environmental information, but potentially also has secondary gaze signaling properties. The modified morphological features of human eyes, increased WHR and white sclera, would be particularly useful for a conspecific presented with a direct gaze. Elongating the human eye and lightening the sclera might suffice to effectively display and amplify gaze direction by providing a more reliable contrast signal in the horizontal plane.

This study also examined averted human and gorilla gazes, in which humans had larger WHR than gorillas. In both humans and gorillas, the amount of visible sclera was larger for averted gazes than direct gazes, but both species displayed similar amounts of sclera overall in both gaze types. The larger amount of sclera displayed in averted gazing gorillas is consistent with the results for averted gazes in Bornean orangutans (Pongo pygmaeus) whose averted gaze was reported to expose as much visible sclera as a direct human gaze [Kaplan and Rogers, 2002]. In this case, the larger SSI of averted gazing gorillas was comparable to direct and averted human SSIs. In other words, an averted gazing gorilla eye displays a similar amount of visible sclera, or more in the case of lowland gorillas, as a direct or averted human gaze.

This species comparison suggests that the morphological changes of the human eye may have developed to specifically enhance the signaling value of direct gazes more than other gaze types. This suggestion is consistent with the current eye gaze literature, which indicates that human infants orient toward and increase their looking times at conspecific eyes that are directed toward them [Farroni et al., 2002]. These results are also consistent with the suggestion that direct gaze may serve special communicative functions, such as signaling communicative intent and displaying and detecting ostension [Gómez, 1996, 2007]. Ostensive communication consists of showing something (e.g., "X") and showing the intent to show something [Gómez, 1996]. This is achieved by drawing another individual's attention to your own attention, as well as any subsequent behaviors, thus displaying communicative intent in this mutual exchange of information. Therefore, direct gaze could work to express and assess intent to engage particular recipients in interaction. Direct gazes can also set the stage for referential communication: they can capture another's attention to oneself and then, through side directed gaze and corresponding gaze following, redirect the addressee's attention to an element in the environment. Understanding ostensive signals may be particularly complex but potentially not unique to humans [see Gómez, 1996, for primates and Téglás et al., 2012, for dogs]. The corresponding morphological changes of the human eye may 
have resulted from the increasing importance of ostensive signaling in humans, in which these particular aspects of the eye amplify the display of direct gazes. Although the results of this study do not support that the elongation of the human eye was accompanied by an increase in the proportion of visible sclera, it is possible that this change may have been accompanied by a white sclera adaptation to enhance iris contrast and facilitate direct gaze communicative functions.

This study also shows that the previous sclera coloration assumptions for human and great ape eyes may require some revision. The natural variability of white sclera in gorillas reported here shows that sclera coloration is not a simple all-or-nothing phenomenon: less than one-third of the gorilla eyes examined were all-dark $(28.0 \%)$. Typically, at least a portion of the sclera offered some degree of potential contrast with the iris. Up to $84.0 \%$ of lowland gorilla eyes possessed some degree of depigmentation, with $11.5 \%$ of averted gazing eyes showing a completely white sclera, "a la human". Additionally, gorillas engaged in averted gazes $(79.4 \%, \mathrm{~N}=94)$ tended to show more depigmentation than those engaged in direct gazes $(55.6 \%, \mathrm{~N}=30)$. In these averted gazes, the greater amount of visible sclera potentially contributes to the visibility of any lighter sclera regions, which possibly makes averted gazes more informative as signals of attention direction in gorillas.

This sclera variability in gorillas also challenges the "gaze camouflaging" function proposed for great ape eyes [Kobayashi and Kohshima, 2001], and we suggest that the function of sclera color is not a simple dichotomy of either amplifying or concealing gaze direction. Interestingly, some of the gorillas displayed a binocular asymmetry of pigmentation. In humans, a single red eye is sufficient to generate the perception of sadness, unhealthiness, and unattractiveness, which indicates that binocular sclera cues are not necessary to make a perceptual judgment of the health or appeal of another individual [Provine et al., 2013a]. This research suggests that when viewing a gorilla possessing asymmetrical sclera, gaze direction could be sufficiently indicated by the depigmented eye. Whether this honest indication of gaze information is beneficial or costly to the individual remains to be investigated. Additionally, the research of Provine et al., 2013 and Provine et al., 2013a, b, demonstrates that the sclera is a variable chromatic medium that plays a reliable secondary role displaying honest biological and social information. Therefore, the evolution of white sclera in humans is potentially the result of multiple selective pressures, including but not exclusive to the development of ostensive communication and as an indicator of health or social/emotional well-being.

In addition to the observed $7 \%$ of gorillas in this study possessing an all-white sclera, other animal species with dark sclera, including dogs, often have white lateral sclera margins [Dziezyc \& Millichamp, 2004]. Therefore, we suggest that when discussing the human eye, the more relevant and uniquely identifying feature is its horizontal elongation and not a uniqueness in sclera color. This elongation, combined with an increased population frequency of the all-white sclera pattern, may have resulted in an optimal device to highlight gaze direction, particularly direct gaze, which would have had important social communicative benefits for early hominins. The selection of the existing white sclera trait in the human lineage may also be related to the emergence of significant, secondary social and biological signaling functions, such as health, reproductive fitness, and emotional state, and should therefore also be considered from this perspective. 


\section{ACKNOWLEDGEMENTS}

The authors thank K. Pullen of the Paignton Zoo and J. Mansell of the Belfast Zoo for their photograph contributions to the project. We would also like to thank D. Leavens, R. Byrne, and two anonymous reviewers for their helpful comments on earlier versions of this manuscript.

\section{CONFLICT OF INTEREST}

The authors declare no conflict of interest.

\section{REFERENCES}

Alley TR. 1980. Infantile colouration as an elicitor of caretaking behaviour in Old World primates. Primates 21:416-429.

Bradley BJ, Mundy NI. 2008. The primate palette: the evolution of primate coloration. Evolutionary Anthropology 17:97-111.

Caro T. 2005. The adaptive significance of coloration in mammals. BioScience 55:125136.

Caro T. 2009. Contrasting coloration in terrestrial mammals. Philosophical Transactions of the Royal Society B 364:537-548.

Changizi MA, Zhang Q, Shimojo S. 2006. Bare skin, blood and the evolution of primate colour vision. Biology Letters 2:217-221.

Dziezyc J, Millichamp NJ. 2004. Color atlas of canine and feline ophthalmology. St. Louis: Elsevier Saunders.

Eckhart G, Lanjouw A. 2008. Mountain gorillas: biology, conservation, and coexistence. Baltimore: Johns Hopkins University.

Emery NJ. 2000. The eyes have it: the neuroethology, function and evolution of social gaze. Neuroscience \& Biobehavioral Reviews 24:581-604.

Farroni T, Csibra G, Simion F, Johnson MH. 2002. Eye contact detection in humans from birth. Proceedings of the National Academy of Sciences USA 99:9602-9605.

Goodall J. 1986. The chimpanzees of Gombe: patterns of behavior. Belknap Press.

Gómez JC. 1996. Ostensive behavior in great apes: the role of eye contact. In: Russon AE, Bard KA, Parker ST, editors. Reaching into thought: the minds of the great apes. Cambridge: Cambridge University Press p. 131-151.

Gómez JC. 2007. Pointing behaviors in apes and human infants: a balanced interpretation. Child Development 78:729-734.

Kaplan G, Rogers LJ. 2002. Patterns of gazing in orangutans (Pongo pygmaeus). International Journal of Primatology 23:501-526.

Knapp S, McCulley JP, Alvarado TP, Hogan RN. 2007. Comparative ocular anatomy of the western lowland gorilla. Veterinary \& Comparative Ophthalmology 10:357362.

Kobayashi H, Hashiya K. 2011. The gaze that grooms: contribution of social factors to the evolution of primate eye morphology. Evolution and Human Behavior 32:157-165.

Kobayashi H, Kohshima S. 2001. Unique morphology of the human eye and its adaptive meaning: comparative studies on external morphology of the primate eye. Journal of Human Evolution 40:419-435.

Laube I, Kamphuis S, Dicke PW, Thier P. 2011. Cortical processing of head- and eye- 
gaze cues guiding joint social attention. NeuroImage 54:1643-1653.

Liang D, Alvarado TP, Oral D, Vargas JM, Denena MM, McCulley, JP. 2005.

Ophthalmic examination of the captive western lowland gorilla (Gorilla gorilla gorilla). Journal of Zoo and Wildlife Medicine 36:430-433.

Little AC, Jones BC, Waitt C, et al. 2008. Symmetry is related to sexual dimorphism in faces: data across culture and species. PLoS ONE 3:e2106.

Norn M. 1985. Pigment spots related to scleral emissaries in Eskimos, Mongols, and Caucasians. Acta Ophthalmologica 63:236-241.

Provine RR, Cabrera MO, Brocato NW, Krosnowski KA. 2011. When the whites of the eyes are red: a uniquely human cue. Ethology 117:395-399.

Provine RR, Cabrera MO, Nave-Blodgett J. 2013a. Binocular symmetry/asymmetry of scleral redness as a cue for sadness, healthiness, and attractiveness in humans. Evolutionary Psychology 11(4):873-884.

Provine RR, Cabrera MO, Nave-Blodgett J. 2013b. Red, yellow, and super-white sclera: uniquely human cues for healthiness, attractiveness, and age. Human Nature 24:126-136.

Provine RR, Nave-Blodgett J, Cabrera MO. 2013. The emotional eye: red sclera as a uniquely human cue of emotion. Ethology 119:993-998.

Schaller G. 1963. The mountain gorilla: ecology and behavior. The University of Chicago Press.

Schmittbuhl M, Minor JML, Allenbach B, Schaaf A. 1999. Shape of the orbital opening: individual characterization and analysis of variability in modern humans, Gorilla gorilla, and Pan troglodytes. Annals of Anatomy 181:299-307.

Téglás E, Gergely A, Kupán K, Miklósi Á, Topál J. 2012. Dogs' gaze following is tuned to human communicative signals. Current Biology 22:209-212.

Tomasello M, Hare B, Lehmann H, Call J. 2007. Reliance on head versus eyes in the gaze-following of great apes and human infants: the cooperative eye hypothesis. Journal of Human Evolution 52:314-320.

Treves A. 1997. Primate natal coats: a preliminary analysis of distribution and function. American Journal of Physical Anthropology 104:47-70.

Zhang P, Watanabe K. 2007. Preliminary study on eye colour in Japanese macaques (Macaca fuscata) in their natural habitat. Primates 48:122-129. 
1 Table I. The overall width/height ratio (WHR) and exposed sclera size (SSI) $( \pm S D)$

2 results for direct and averted gazes by species. * indicates that significant differences

3 were observed between direct and averted gazes for that particular species. $N=$ the

4 number of individuals measured.

\begin{tabular}{lllllll}
\hline & Humans & & \multicolumn{2}{l}{ Lowland gorillas } & \multicolumn{2}{l}{ Mountain gorillas } \\
& WHR $(N)$ & SSI $(N)$ & WHR $(N)$ & SSI $(N)$ & WHR $(N)$ & SSI $(N)$ \\
\cline { 2 - 7 } Direct gaze & $3.24 \pm 0.49 *$ & $1.82 \pm 0.13^{*}$ & $2.02 \pm 0.29 *$ & $1.64 \pm 0.35^{*}$ & $1.86 \pm 0.29$ & $1.67 \pm 0.25$ \\
& $(13)$ & $(13)$ & $(21)$ & $(17)$ & $(8)$ & $(7)$ \\
Averted gaze & $2.94 \pm 0.40$ & $2.07 \pm 0.16$ & $2.21 \pm 0.28$ & $2.21 \pm 0.47$ & $1.77 \pm 0.19$ & $1.95 \pm 0.35$ \\
& $(13)$ & $(13)$ & $(33)$ & $(29)$ & $(16)$ & $(16)$ \\
\hline
\end{tabular}


5 Table II. The frequencies of white sclera patterns observed in the eyes of direct and 6 averted gazing mountain and lowland gorillas. The values represent the number of eyes 7 observed with a particular sclera pattern.

\begin{tabular}{lllll}
\hline \multirow{2}{*}{ Pattern } & \multicolumn{2}{l}{ Lowland gorillas } & \multicolumn{2}{l}{ Mountain gorillas } \\
& Direct & Averted & Direct & Averted \\
\hline All-dark & 12 & 7 & 12 & 17 \\
Patchy & 7 & 17 & 1 & 10 \\
Banded & 3 & 16 & - & 6 \\
Crescent & 10 & 17 & 1 & 4 \\
Combination & 6 & 12 & - & 2 \\
All-white & 2 & 9 & - & 1 \\
\hline
\end{tabular}


8 Fig. 1 - Gorillas with the white sclera characteristic: A) Nadia, Madrid Zoo, Madrid, 9 Spain (J-C Gómez) and B) Bana, Brookfield Zoo, Brookfield, IL (J. Mayhew).

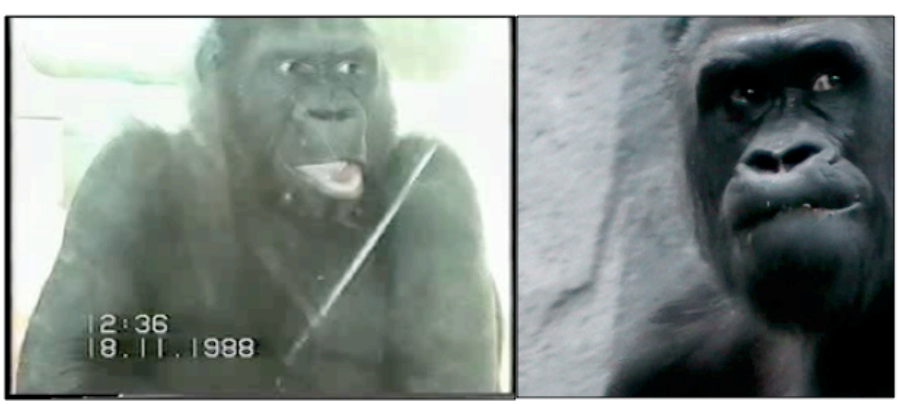


11 Fig. 2 - The measurements A-D were obtained for each eye: top, width/height ratio (A/B) 12 and bottom, exposed sclera size (C/D).

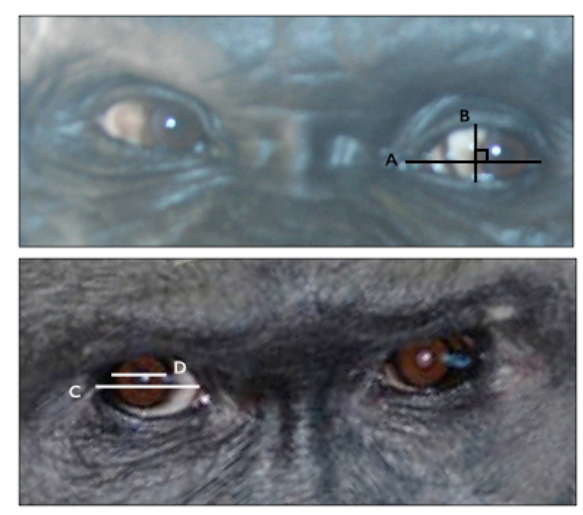


14 Fig. 3 - Sclera patterns: 1) all-dark sclera (score of 0); 2) patchy (score of 7); 3) banded 15 (score of 6); 4) crescent (score of 6); and 5) all-white (score of 10). A combination of 16 patterns may also be observed, and patterns may vary in the left and right eyes of the 17 individuals.

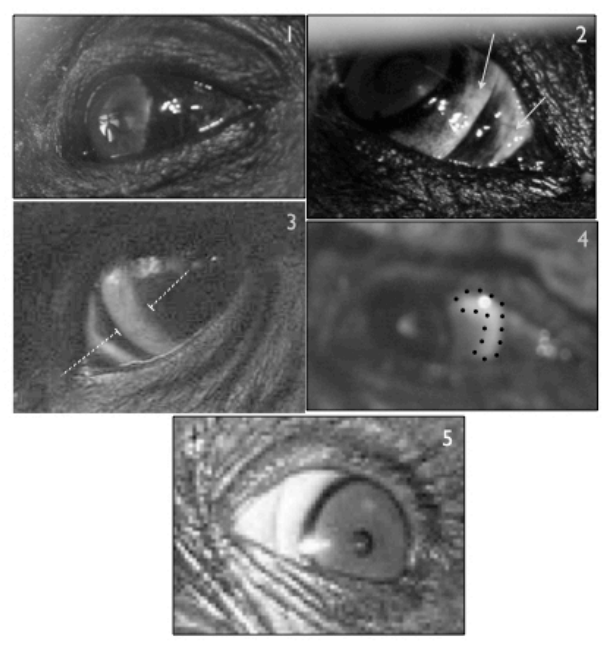


19 Fig. 4 - The amount of depigmentation observed in direct gazing humans, mountain 20 gorillas, and lowland gorillas.

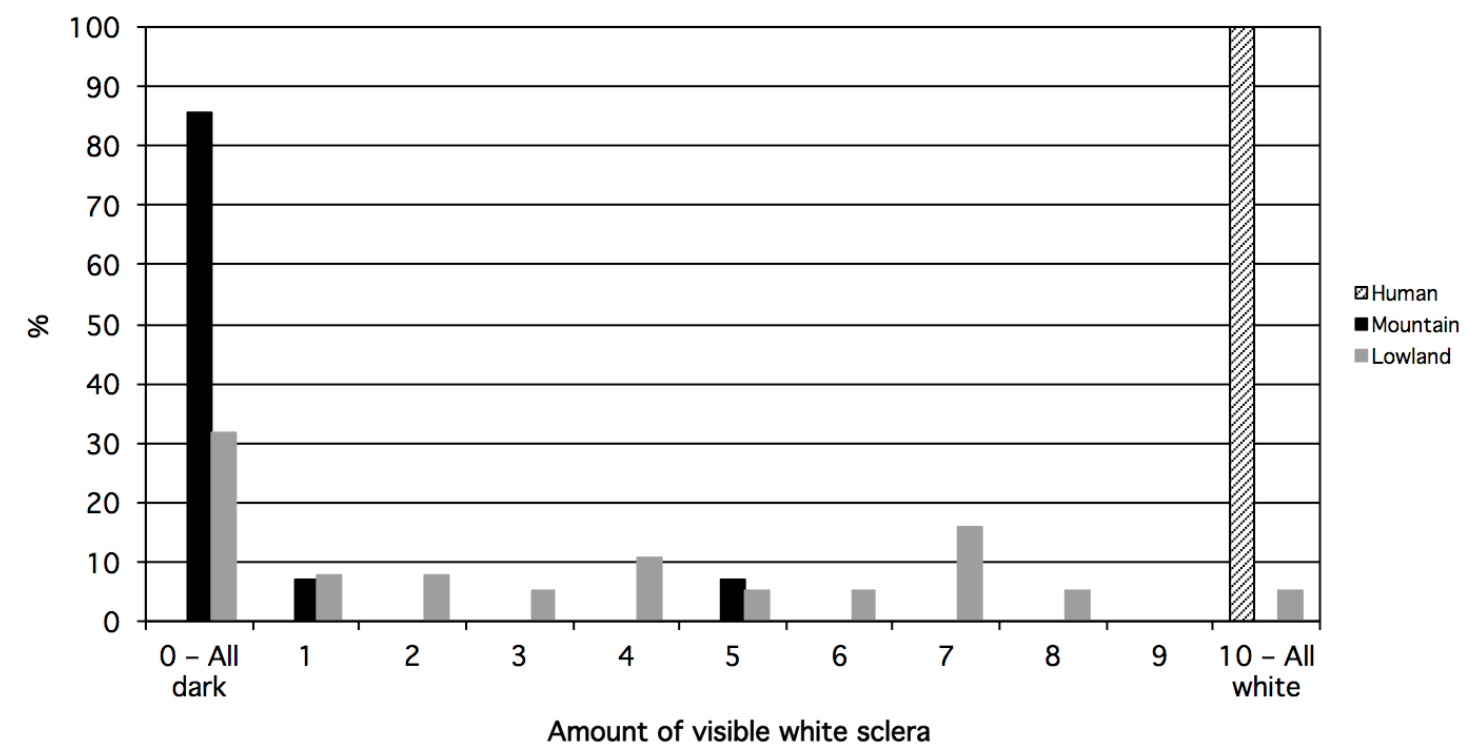


22 Fig. 5 - The amount of depigmentation observed in averted gazing humans, mountain 23 gorillas, and lowland gorillas.

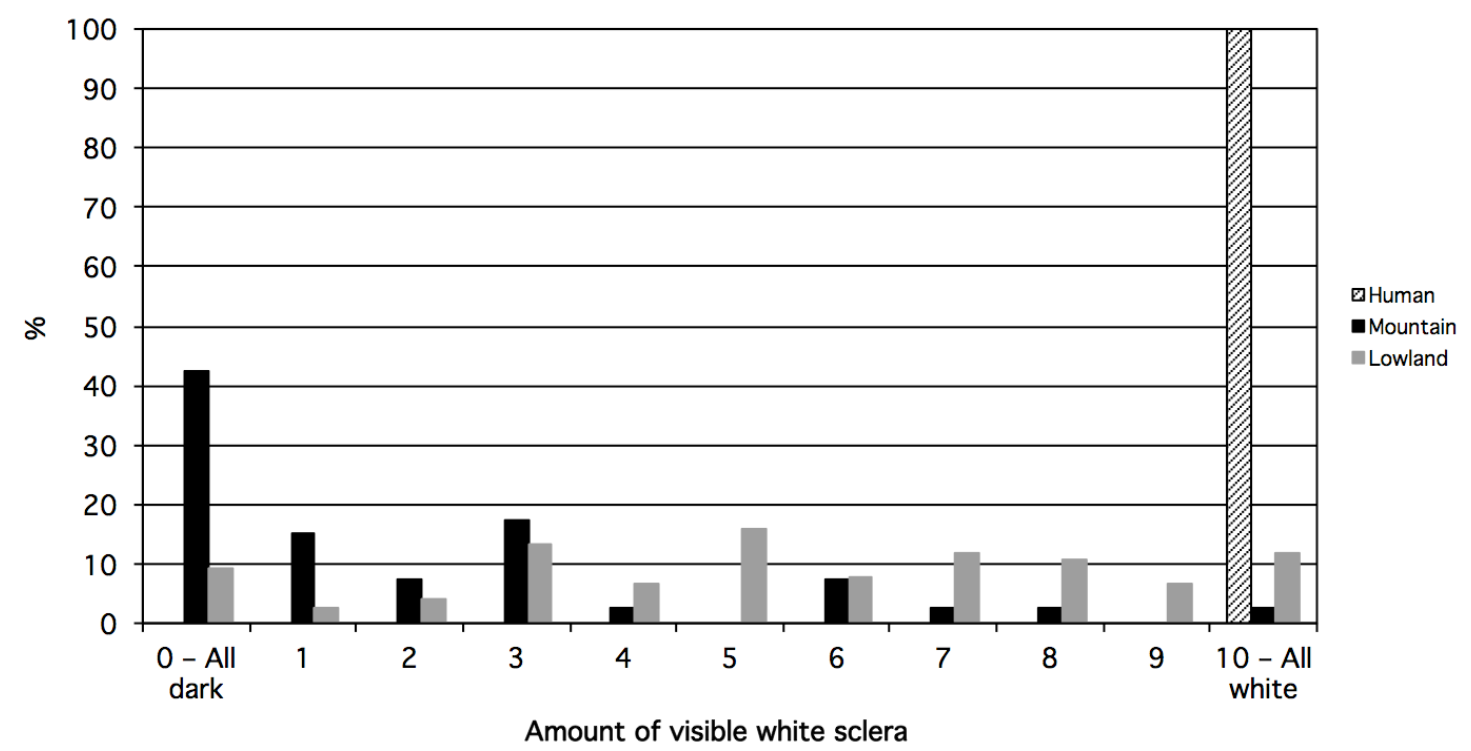

